\title{
Imaging technologies for preclinical models of bone and joint disorders
}

\author{
Jordi L Tremoleda ${ }^{1 *}$, Magdy Khalil1', Luke L Gompels², Marzena Wylezinska-Arridge', Tonia Vincent ${ }^{2}$ and \\ Willy Gsell ${ }^{1}$
}

\begin{abstract}
Preclinical models for musculoskeletal disorders are critical for understanding the pathogenesis of bone and joint disorders in humans and the development of effective therapies. The assessment of these models primarily relies on morphological analysis which remains time consuming and costly, requiring large numbers of animals to be tested through different stages of the disease. The implementation of preclinical imaging represents a keystone in the refinement of animal models allowing longitudinal studies and enabling a powerful, non-invasive and clinically translatable way for monitoring disease progression in real time. Our aim is to highlight examples that demonstrate the advantages and limitations of different imaging modalities including magnetic resonance imaging (MRI), computed tomography (CT), positron emission tomography (PET), single-photon emission computed tomography (SPECT) and optical imaging. All of which are in current use in preclinical skeletal research. MRI can provide high resolution of soft tissue structures, but imaging requires comparatively long acquisition times; hence, animals require long-term anaesthesia. CT is extensively used in bone and joint disorders providing excellent spatial resolution and good contrast for bone imaging. Despite its excellent structural assessment of mineralized structures, CT does not provide in vivo functional information of ongoing biological processes. Nuclear medicine is a very promising tool for investigating functional and molecular processes in vivo with new tracers becoming available as biomarkers. The combined use of imaging modalities also holds significant potential for the assessment of disease pathogenesis in animal models of musculoskeletal disorders, minimising the use of conventional invasive methods and animal redundancy.
\end{abstract}

Keywords: imaging, animal models, bone, cartilage, micro-CT

\section{Introduction}

Bone and joint disorders impose an enormous social and economic burden on society, causing disability and substantial patient morbidity. There is a rising demand for developing effective therapies to improve such conditions [1]. The process of discovering and bringing drugs to the clinics remains long and expensive; thus, improving its efficiency remains a major target. Preclinical testing plays a major role in this process enabling powerful and clinically translatable methods for monitoring disease progression and testing drug candidates. Imaging is becoming an important key technology in

\footnotetext{
* Correspondence: jordi.lopez-tremoleda@imperial.ac.uk

${ }^{1}$ Biological Imaging Centre (BIC), Medical Research Council (MRC) Clinical Sciences Centre, Imperial College London, Hammersmith Campus, London W12 ONN, UK

Full list of author information is available at the end of the article
}

this process, with its ability to deliver non-invasive and quantitative cellular and molecular information that can access the mechanisms of drug action. Thus, imaging is one of the most promising technologies for improving the drug development process, facilitating translation between preclinical and clinical findings [2]. In the last few years, many large pharmaceutical companies and research institutions are implementing imaging at critical stages of their preclinical studies.

With advances in transgenics and animal models for human disease, researchers are increasingly using imaging not only for drug discovery but also for phenotyping and understanding the pathophysiology of disease. Its use has represented a keystone in the refinement of animal models, allowing longitudinal studies and enabling a powerful, non-invasive and clinically translatable way for monitoring disease progression in real

\section{SpringerOpen ${ }^{\circ}$}

(c) 2011 Tremoleda et al; licensee Springer. This is an Open Access article distributed under the terms of the Creative Commons Attribution License (http://creativecommons.org/licenses/by/2.0), which permits unrestricted use, distribution, and reproduction in any medium, provided the original work is properly cited. 
time. For many years, studies in animal models relied on histological analysis of tissues and/or organs post-mortem. These destructive methods limited the ability of researchers to study the progression of the disease on a single animal serially over time as well as assessing therapeutic efficiency overtime.

Imaging technologies provide good tools for assessing anatomical, morphological, physiological and functional parameters and molecular and cellular processes in animal models of disease. Imaging protocols and agents have been developed to enable good spatial resolution for the physiological and functional properties of targeted tissue, including blood flow, tissue permeability, metabolism, tissue density, cellular proliferation and oxygenation. Currently, imaging is increasingly being implemented at the late stage of preclinical development through to the clinical phases, taking a major role in the validation of specific candidate drug or treatment regime. These non-invasive technologies allow for the combined use of different modalities to obtain multiple physiological and functional parameters from a single animal study. This optimizes greatly the power of such animal studies and the efficacy of experimental readouts.

The objective of this review is to familiarise the reader with a selection from the range of imaging possibilities that are available for assessing bone and joint disorders in animal models. We will review the technologies available, discuss their current applications and address their challenges and future implications for refinement of musculoskeletal animal models.

\section{Imaging technologies \\ Micro-computed tomographic imaging \\ Overview of CT technology}

The development of dedicated imaging equipment for small animals and, in particular, the implementation of computed tomography (CT) have revolutionised the use of animal models in musculoskeletal research, becoming the gold standard for evaluation of bone morphology and micro-architecture in animal models $[3,4]$. While histomorphometric assessment has been extensively used as the main standard for investigating bone architecture, the development of 3D imaging techniques such as CT have provided an accurate non-invasive tool for directly measuring bone architecture. Indeed, since the development of clinical CT, the examination of small animals for research using purpose built CT has rapidly advanced providing high-quality resolution and fast reconstruction and assessment protocols for preclinical applications [5-7].

Micro-CT uses X-ray attenuation data acquired at multiple viewing angles to reconstruct a 3D representation of the imaged specimen, characterising the spatial distribution of the material density $[8,9]$. Currently available micro-CT scanners can achieve high resolution with an isotopic voxel size of as low as a few micrometres (down to $5 \mu \mathrm{m}$; although new generation on nano-scanners can go down below $1 \mu \mathrm{m}$ ) [10]. There are, broadly speaking, two different micro-CT construction systems, one type in which the examined object is placed in the centre and the $\mathrm{X}$-ray detector and radiation source is mounted in a gantry that rotates around it; in this system, the geometrical magnification is defined by the source-detector distance. This set-up is the one most commonly adapted for animal scanners. In the second type of CT scanner, the object is rotated within the course of the X-ray beam and the set-up allows the free positioning of the sample between the detector and the source, allowing the adjustment of the magnification level. This second construction is more often utilised in ex vivo custom-built systems. There are also differences on the beam geometry of the X-ray source used. Images can be acquired by using either a fan-shaped beam in which data are acquired through dynamic acquisition plane by plane or by a cone beam this is also called 'volume-CT' [11] where the scanned subject is captured completely (based on the axial extent of the CT field of view) in one rotation, speeding up the imaging process. Furthermore, micro-CT systems can be fitted with a flat-panel-based detector system with slip ring technology that allows for very high-speed data collection [12]. The rapid acquisition times come at the expense of compromising spatial resolution, but this may be justifiable for in vivo applications that require rapid scan times such as perfusion imaging and highthroughput imaging. Similarly, tissue contrast enhancement can be induced by using a dual-energy X-ray computed tomography method in which the projection data are acquired by using two different X-ray spectra [13]. Figure 1 illustrates the different micro-CT systems technologies.

Current availability of multi-modality imaging platforms which can provide integrated positron emission tomography (PET)/single-photon emission computed tomography (SPECT)/computed tomography (CT) imaging and analysis are extremely useful for co-registering images within the same gantry, facilitating a multimodal imaging approach within the same animal in vivo at the same time points during disease evolution [14]. Moreover, rapid image acquisition can also be facilitated by gating signal acquisition to cardiac and respiratory cycles by utilising a high-speed shutter system that allows image times as short as $10 \mathrm{~ms}$ [15].

Micro-CT imaging applications of preclinical models for bone and joint disorders

Micro-CT is extensively used to investigate the structure and density of bone in rodents. Micro-CT has high spatial resolution and contrast for imaging mineralised 


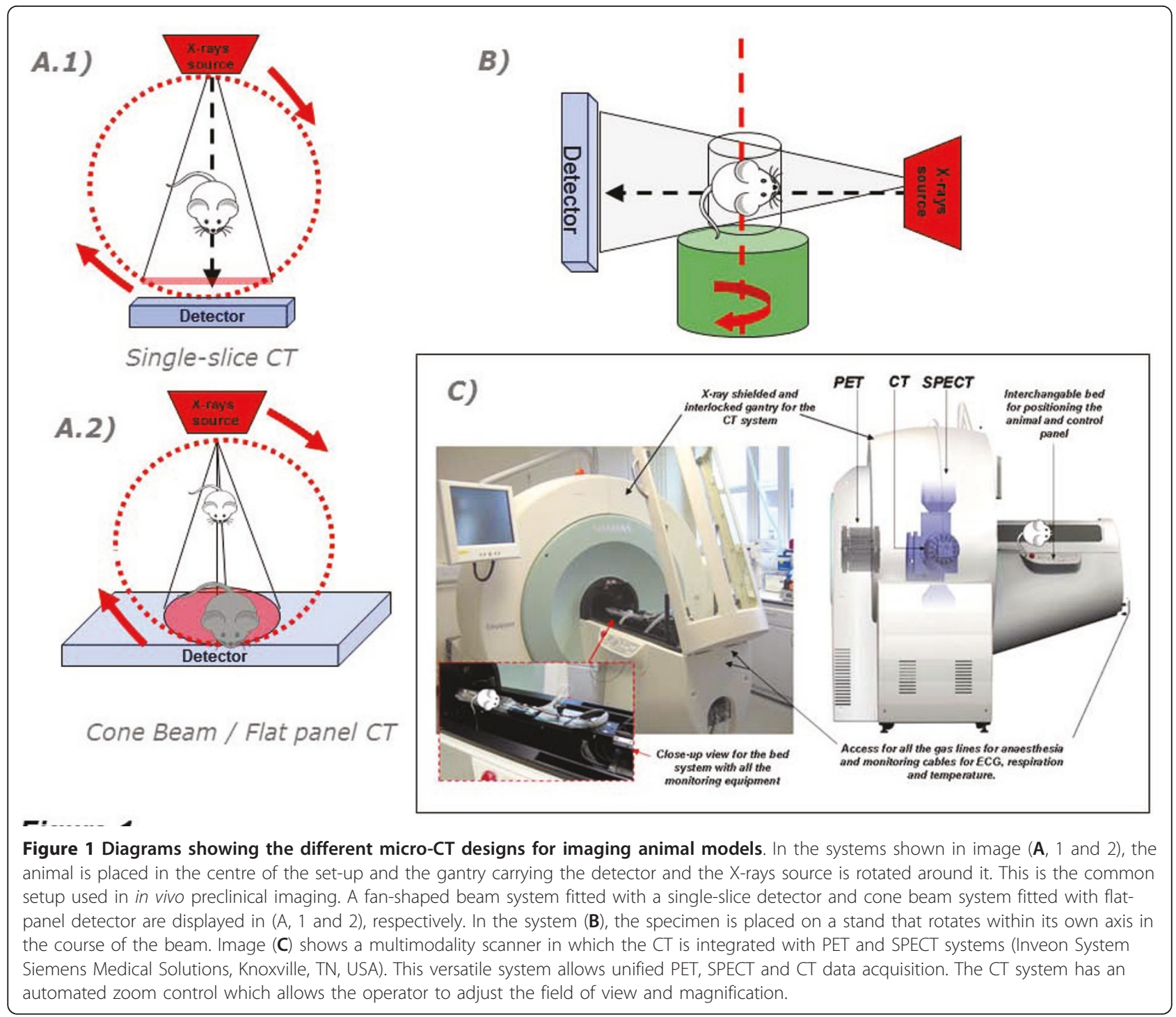

tissues and the ability to qualitatively and quantitatively assess 3D bone structures. This allows measurements of trabecular morphology such as thickness and separation. Micro-CT has been used for a wide range of bone studies, including bone anatomy and density to assess bone repair during fracture healing [16]; bone resorption, remodelling and regeneration [17]; bone neoplasm and metastases [18] and bone changes influenced by metabolic disorders, e.g. osteoporosis [19] and the characterisation of skeletal phenotypes from different mouse strains [20]. Figure 2 provides an example of different imaging acquisitions of various mouse bones used for skeleton phenotypes and bone repair studies.

The high isotropic resolution can also provide good information on trabecular bone spatial orientation patterns, density, and geometry and growth plate morphology. While much finer detailed imaging can be achieved in ex vivo samples, micro-CT imaging has proven very valuable for in vivo longitudinal studies. At least a 100 $\mu \mathrm{m}$ isotropic spatial resolution can be effectively achieved within a safe and reasonable acquisition time through longitudinal in vivo studies [21]. Some studies have reported in vivo isotropic resolutions down to 15 $\mu \mathrm{m}$ in serial imaging sessions using rats (seven sessions of 10 min acquisitions with an absorbed dose of 0.5 GyCTDI) [22], but there are concerns about the amount of ionizing radiation delivered during repetitive in vivo scanning. This radiation may introduce unwanted effects on the tissues or processes of interest or have an adverse effect on animal welfare [23].

Micro-CT imaging has also proven to be very valuable in investigating morphometric changes in joints in osteoarthritis (OA) animal models [24-26]; it has been successfully applied to study changes in the subchondral 


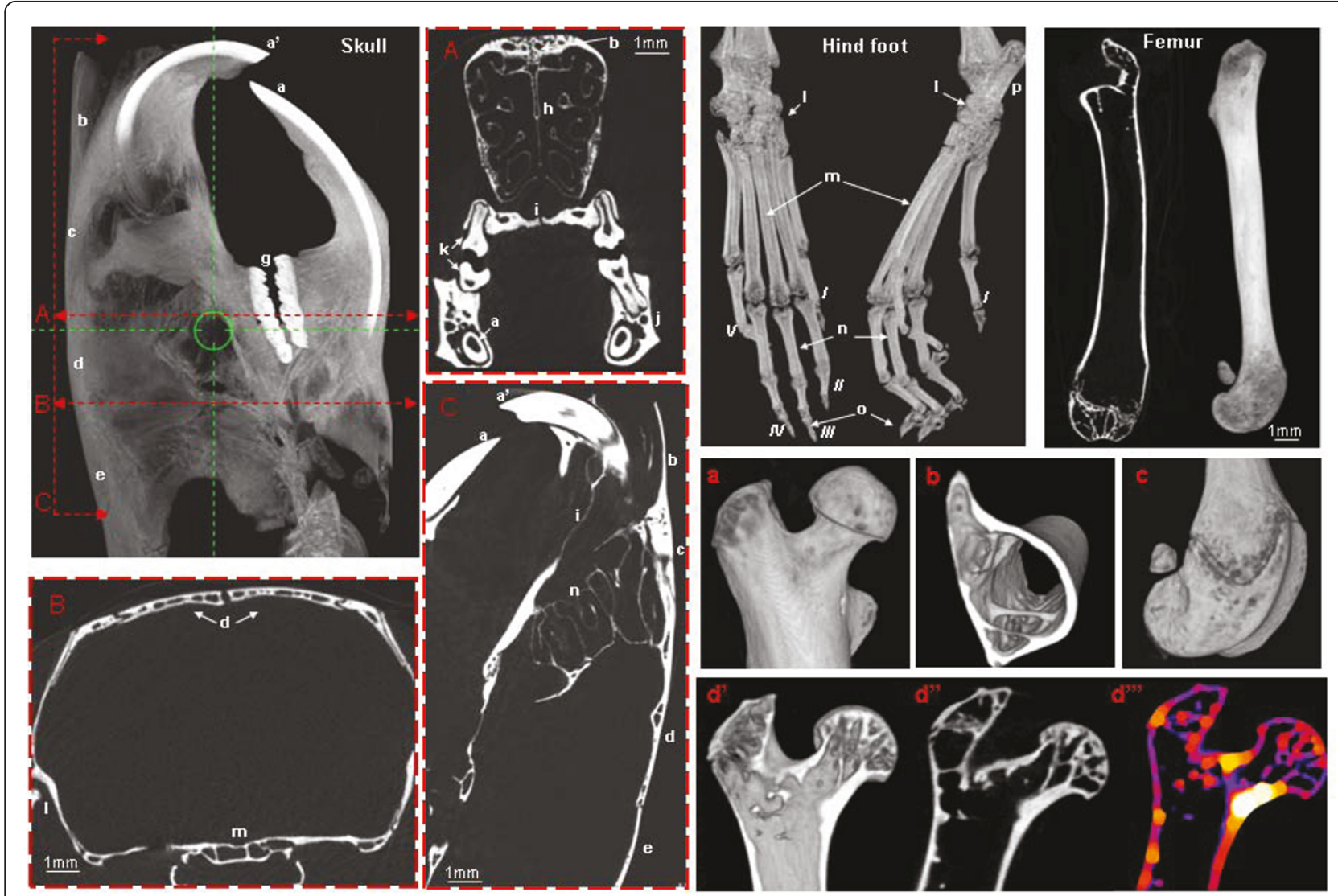

Figure 2 Micro-CT images acquired ex vivo from Nude BALB/c mouse skull (3D-volume rendering image). Displaying different anatomical regions: (a, $\mathrm{a}^{\prime}$ ) lower and upper incisor tooth, (b) nasal bone, (c) frontal bone, (d) parietal bone, (e) intraparietal bone, (f) right mandible and ( $\mathrm{g}$ ) molar tooth. (A, B) Coronal views of cranial and caudal 34 areas of the skull, respectively, displaying in image A: (a) roots of the lower incisor, (b) frontal bone, (h) nasal septum, (i) maxillar/palatine bone, (j) mandible and ( $k$ ) roots of molar tooth; in B: (d) parietal bones, (I) temporal line and ( $m$ ) basis sphenoid bone. (C) Sagittal view of the skull displaying incisor roots (a, $\left.a^{\prime}\right)$, nasal, frontal, parietal, intraparietal bones (b, $\left.c, d, e\right)$ and endoturbine structures (n). Micro-CT images from Nude BALB/C mouse hind foot (3D-volume rendering) showing the I-V phalanges, (I) tarsocrural joint, (m) metatarsal bone, $(n)$ digital bone, (o) claw, (p) calcaneous bone. Examples of micro-CT C57BL/6 mouse femur showing coronal view and 3D-volume rendering image. (a, b, c) Close views of the proximal epiphysis (head and greater trochanter), metaphysis area displaying cortical and trabecular bone and the femur condyles, respectively. (d) Images displaying a sagittal 3D and 2D cross section of the proximal epiphysis $\left(d^{\prime}, d^{\prime \prime}\right)$ and a sagittal cross view showing a map of the bone thickness with higher density areas displayed as white (femoral neck). All the samples were acquired at $80 \mathrm{kVp}, 500 \mu \mathrm{A}$ and with a pixel size of $9.5 \mu \mathrm{m}$; images were reconstructed in Hounsfield units (HU) and processed with ImageJ (NIH, Bethesda, MD, USA).

bone architecture in excised osteoarthritic knees in collagenase-induced OA mice [27] and in surgical destabilisation models [28]. Figure 3 shows micro-CT athrograms of mouse knees from the surgical destabilisation of the medial meniscus (DMM) model. Recently, Stok and collaborators [29], by imaging excised knees ex vivo, showed how changes in subchondral bone seem to be inversely correlated to the ongoing degenerative changes in the articular cartilage in STR/ort mice (naturally occurring $\mathrm{OA}$ ). Indeed, micro-CT imaging provides quantitative and qualitative $2 \mathrm{D}$ and $3 \mathrm{D}$ assessments of bony structures in the osteoarthritic joint such as subchondral bone morphology and bone mineral density, trabecular bone patterns, meniscus morphology, heterotopic ossification and subchondral cyst formation [30]. Figure 3 shows micro-CT athrograms of mouse knees in C57BL/6 mice, 8 weeks after surgical destabilisation of the medial meniscus to investigate changes in the subchondral bone (Tremoleda et al., unpublished data). Interestingly, such technology has also been successfully incorporated to monitor the progression of OA in longitudinal studies in rats [31].

One of the major challenges for imaging osteoarthitis models is that the micro-CT has low sensitivity for soft tissue; hence, compromising the visualisation of the degenerative changes in the articular cartilage. Contrast agents can be used to enhance the contrast resolution of CT imaging, and there have been a few attempts to indirectly 


\section{Micro-CT images of the knees in a mouse model of osteoarthtiris (DMM model)}
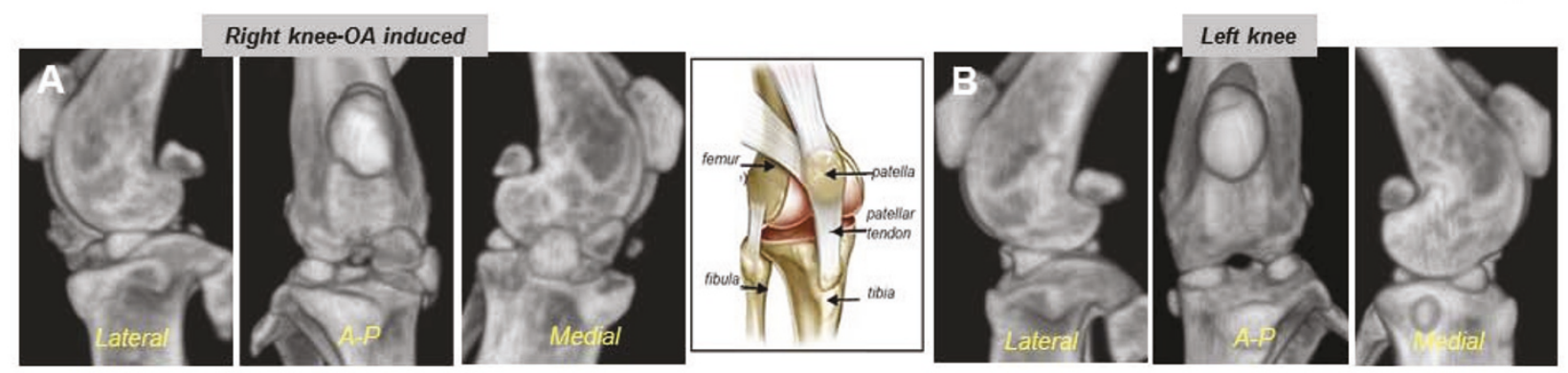

\section{Bone density maps}

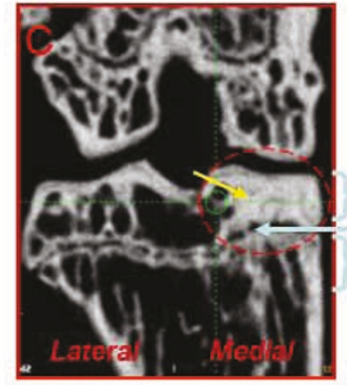

Coronalview

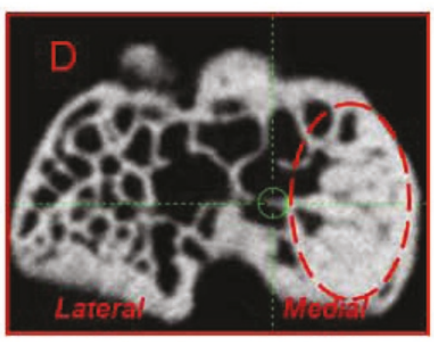

Transverse view
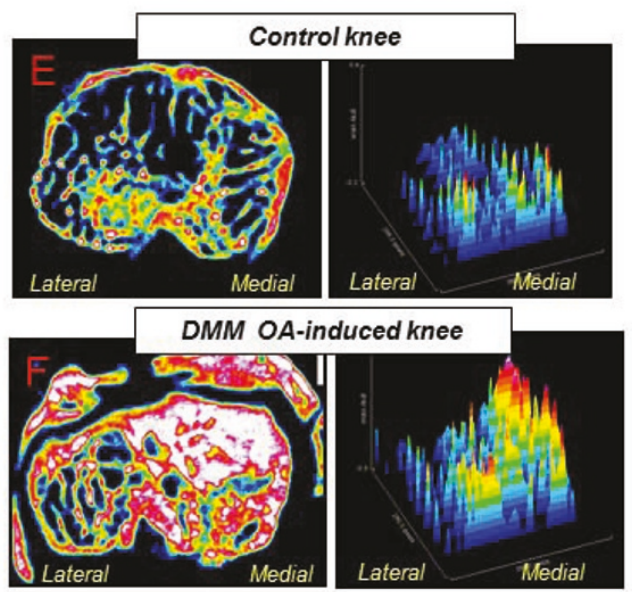

Figure 3 Computed tomography imaging of the knees in a mouse model of osteoarthritis (DMM model). Images of non-injured mouse knee (left knee, B) and 2 months after surgical induction of osteoarthritis (DMM model; right knee, A) were acquired ex vivo at $80 \mathrm{kVp}, 500 \mu \mathrm{A}$ and with a pixel size of $35 \mu \mathrm{m}$. Series (A) and (B) show 3D surface rendering CT lateral, anterior/posterior and medial views of the DMM-knee displaying OA-related derangements of the knee morphology 35 (remodelling of subchondral bone, hypertrophic calcifications; A), and of a noninjured knee (B), respectively. Images were analysed for differences in subchondral sclerosis in the epiphysis: (C) coronal and (Dtransverse views. Bone density maps show different densities throughout the subchondral weight-bearing lateral and medial regions of the tibial plateau in a controlled non-injured knee $(\mathbf{E})$ versus a DMM OA-induced knee $(\mathbf{F})$. Notice the high-density area in the medial region (DMM-injury site). Bone density was measured from micro-CT images (voxel size of $35 \mu \mathrm{m}$ ) normalise to $\mathrm{HU}$ units, and images were processed with Image $(\mathrm{NIH})$.

visualise the cartilage morphology using these agents in ex vivo samples [32,33]. To compensate for the poor radioopacity of the cartilage, tissues to be imaged are equilibrated with an ionic contrast agent which is taken up by the cartilage matrix and distributed inversely to the density of the negatively charged glycosaminoglycans (GAGs). This contrast-enhanced CT technique which is based on the detection of the equilibrium partitioning of an ionic contrast agent (EPIC-micro-CT) provides good 3D characterisation of the articular surface morphology and ligament insertions in situ in ex vivo rabbit knees [31]. This technique allows for the monitoring of surface contours and healing processes in injured articular cartilage in defect repair studies as well as changes in cartilage thickness. Changes to other anatomical features such as ligaments and non-calcified menisci can also be detected. While the use of this methodology has great potential for assessing cartilage degeneration in in vivo longitudinal studies, the thin layer of articular cartilage and the small joint space in mice combined with diffusion variability between contrast agents remain challenges for accurate quantification of signal change.

\section{Magnetic resonance imaging}

\section{Overview of the MRI technology}

Magnetic resonance imaging (MRI) is a non-ionizing 3D imaging technique that has advantages over other methods that depend on ionizing radiation such as CT, SPECT and PET. It remains one of the main imaging applications of choice for assessment of musculoskeletal tissue structures such as tendons, cartilage, menisci and ligaments in the clinical setting and has also being successfully tested in animal models (mice [34], rats [35] and rabbits [36]). 
MRI technology uses the magnetic properties of atoms and molecules of tissues to be imaged and their interactions with both a large external magnetic field and radiowaves. The proton ${ }^{1} \mathrm{H}$ is the nucleus mostly used for anatomical imaging because of its abundance of soft tissue structures where there is a high water content. MRI technology utilises a powerful magnetic field (nonionizing radiation) to align the nuclear magnetization of hydrogen atoms, and then, radio frequency (RF) pulses (through RF coils) are used to systematically alter this alignment, causing the hydrogen nuclei to produce a rotating magnetic field that is detectable by the scanner. The application of radio frequency pulses rotates the magnetization by $90^{\circ}$. Then, the magnetization returns to its initial value, and the rate at which the magnetization decays away is characterised by two relaxation times named as T1 and T2. These signals are then digitalised and processed to build up enough information to construct an image of the targeted body area. It is this relationship between field strength and frequency implemented onto the hydrogen atoms within the tissue that allows the use of nuclear magnetic resonance for imaging.

\section{MRI applications for imaging preclinical models for bone and joint disorders}

MRI assessment of bone structure has remained a challenge due to its composite biomaterial characteristics. Bone is made up of an organic substrate (mostly collagen type I approximately $40 \%$ by volume) and mineral crystals of hydroxyapatite (approximately 45\%). The remaining volume is occupied by water (approximately $15 \%)$. Due to this limited water composition, proton imaging of bone remains challenging. Advanced microMRI systems have been used successfully to image trabecular bone in rats in vivo [37]. These methods rely on indirectly imaging the trabecular bone structure that appears as a signal void surrounded by high-intensity signal from the fatty bone marrow. Because of the small trabecular thickness in small animals (50 to $100 \mu \mathrm{m}$ in rats; 40 to $60 \mu \mathrm{m}$ in mice), the resolution requirements are more stringent with a high risk of overestimation of the trabecular thickness depending on the volume of the trabeculae and therefore an adverse signal-to-noise ratio - reinforcing the need for longer acquisition times.

In the preclinical setting, the acquisition of high-resolution MRI within an acceptable time frame remains an important drawback due to the relatively small size of anatomical structures in rodents; long acquisition is needed to generate sufficient isotropic (3D) resolution with a small-bore MRI and with high field strength. This needs to be performed with the animal under general anaesthesia.

To overcome the limited detection sensitivity and to increase the signal-to-noise ratio, enhanced pulse sequences have been developed. These allow adequate sample volumes to be scanned with scan times maintained within the limits of in vivo tolerance. 3D gradient-echo sequences allow for a shorter TE than spinecho or fast spin-echo sequences, allowing for faster data acquisition compared to fast spin-echo sequences, and therefore allow more data averaging within a fixed duration of scan time [38].

One of the most critical aspects of preclinical MRI is the radio frequency coil which drives both the signal excitation and reception. Because the coil's sensitivity increases as its volume decreases, animal coils are smaller than humans, improving the signal gain significantly while still scanning the anatomical area of interest. Takahashi and collaborators [39] successfully applied MRI technology using a 3D spin-echo pulse sequence in conjunction with spectroscopy to investigate the longitudinal changes in trabecular bone architecture in response to pharmacological interventions in rabbits. Another promising approach is based on the use of ultra-short echo-time (UTE) radial acquisition sequences with hard pulse excitation, which can detect subtle changes in mineral phosphorus and water contents of cortical bone $\left({ }^{31} \mathrm{P}\right.$ and $\left.{ }^{1} \mathrm{H}\right)$, as applied for investigating changes in bone mineralization in ex vivo bones from oestrogen deficiency-induced ovariectomised rats [40]. The quantification of water content through UTE-MRI provides a good technique for measuring bone's hydration state in situ with great potential for investigating bone metabolic disorders in vivo. Figure 4 displays the magnetic resonance (MR) images of rat and mice knees obtained ex vivo in a 9.4-T MRI scanner using a 3D gradient echo and fast spin-echo multi-slice acquisitions, respectively.

MRI is extensively used in clinics for assessment of articular cartilage in joint disorders. As in clinical MRI, the MR sequence that best delineated the cartilage from the surrounding tissues, i.e. 3D fat-suppressed spoiled gradient echo, has also been successfully applied for depicting changes in the rat knee of OA models [41]. Moreover, some studies in ex vivo rat knees have measured the spatial distribution of $\mathrm{T} 2$ relaxation times as a function of the water content and the collagen ultrastructure correlating with the structural integrity of the articular cartilage [42]. These measurements may permit early detection of changes of cartilage matrix integrity, which could lead to osteoarthritis, and thus would be a relevant preclinical model for the development of clinical treatment before any cartilage morphological alterations occur.

Another commonly used approach is the use of contrast agents such as gadolinium (Gd-DTPA, gadopentetate dimeglumine or Magnevist ${ }^{\mathrm{TM}}$ Schering, Berlin, Germany). The Gd-DTPA penetrates into cartilage and 


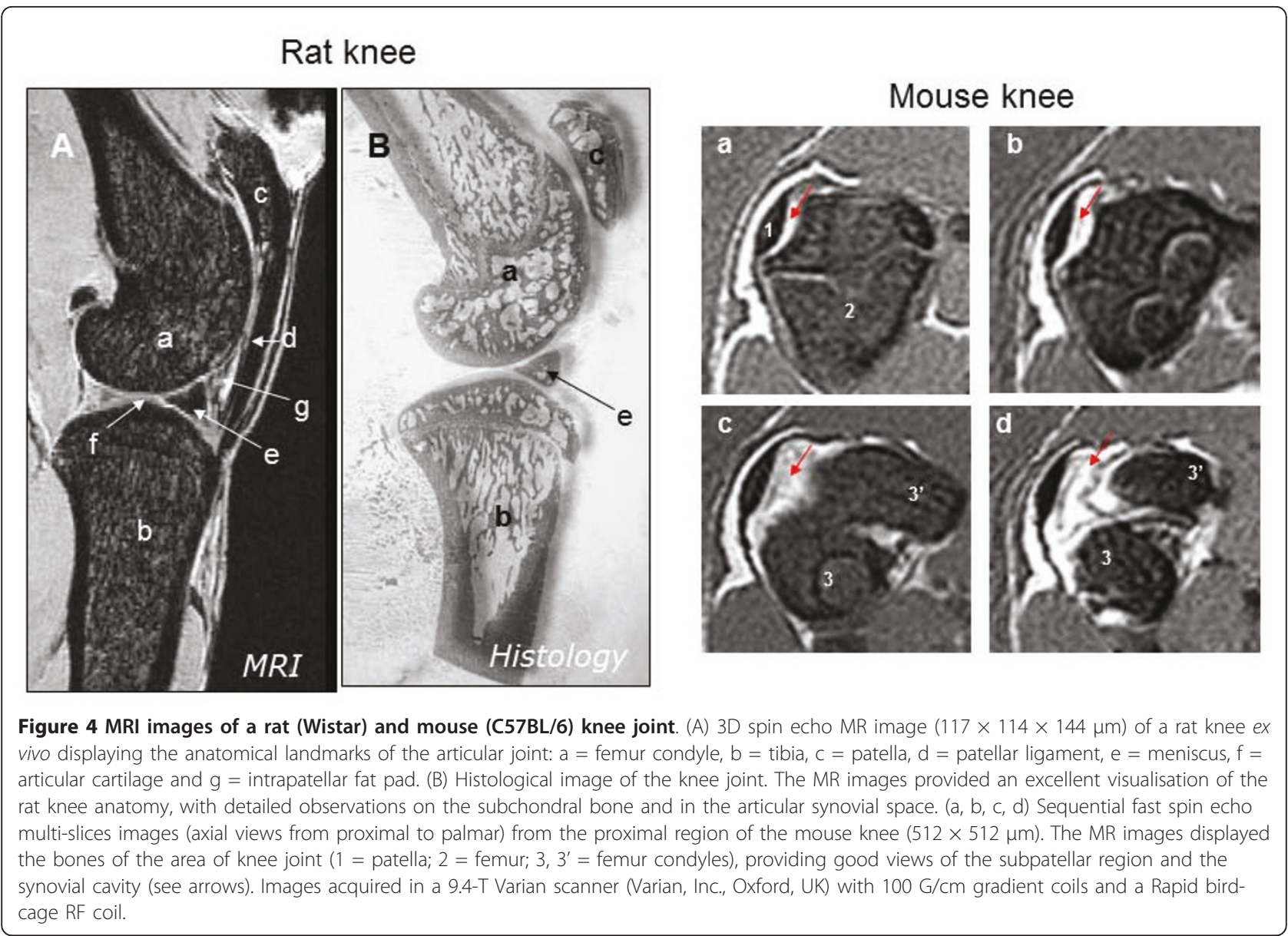

distributes preferentially to areas of the cartilage in which the GAGs are low. Subsequently, this will induce a decrease in T1 relaxation that reflects the Gd-DTPA concentration and thus the distribution of GAGs. This clinically validated technique is referred to as delayed gadolinium-enhanced MRI of cartilage and has also been investigated in preclinical models [43]. Another application of contrast-enhanced MRI is the assessment of blood flow perfusion and permeability which has important physiopathological relevance as reduced bone blood perfusion is also an indicator of disease progression and severity. Contrast agents like gadolinium (Gd) have been applied for imaging bone blood flow using dynamic contrast-enhanced MRI techniques in animal models of OA [41]. These techniques may be very valuable to assess the healing process and estimate the risk of avascular necrosis.

Finally, ongoing developments in preclinical MRI technology, with the implementation of phased-array coils which by contrast with the traditional single-channel surface coil can increase the signal-to-noise ratio, thereby providing superior image sensitivity covering a specific field of view, have been successfully used for imaging the knee joint in rats [44].

\section{Nuclear imaging technologies \\ PET/SPECT systems technology}

Bone scintigraphy is extensively used as one of the most common diagnostic techniques to investigate bone lesions and metastases in various musculoskeletal conditions and in diagnostic orthopaedic medicine. Further innovations such as single-photon emission computed tomography (SPECT) and positron emission tomography (PET) have allowed the acquisitions of whole-body images of the entire skeleton. They provide increased sensitivity for lesion detection and, importantly, a 3D localization of the radiation emitted by radionuclide imaging agents or biomarkers with very high detection sensitivity down to nano- or picomolar concentration.

The growth in clinical nuclear imaging applications has led to the development of SPECT and PET scanners dedicated for small animal imaging [45,46]. These systems have to cope with the small size of rodents; hence, they have to achieve enhanced spatial resolution and 
high sensitivity for the targeted biomarker. The application of this technology in preclinical models has a significant scope for non-invasively studying dynamic biological processes at the molecular and cellular level. It provides good functional information through the detection of onset and progression of a given biological process, and can assist in the development of biomarkers and in measuring the effectiveness of new treatments.

SPECT systems record gamma rays directly after radionuclide emission. The system uses a gamma camera to acquire projections data that are detected using a parallel hole collimator. However, most of the preclinical SPECT scanners are equipped with multipinhole collimators to acquire high spatial resolution of foci of gamma-emitting tracers within the subject volume. Then, a tomographic reconstruction of the data is acquired yielding a 3D dataset that can then be manipulated to show any particular axis of the body. PET systems also detect gamma rays that are emitted by a biomarker tracer labelled to a positron emitter (F-18, C11, N-13, O-15). These radio-nuclides emit positrons which cause two gamma photons to be emitted in opposite directions by annihilation with an electron. The two photons are accepted to be in coincidence if their incidence on a detector pair is within a predefined timing resolution window. The scanner detects these dual emissions 'coincident in time', providing a higher radiation on a given location and thus higher resolution images. This is in contrast to SPECT systems that rely on hardware collimation. The resolution of preclinical PET scanner lies in the range of 1 to $2 \mathrm{~mm}$, but some stateof-the-art dedicated preclinical SPECT systems can provide better resolution capabilities (down to the submillimetre range) [43]. SPECT radiopharmaceuticals have a long half-life, and they are routinely produced for clinical nuclear medicine, making SPECT isotopes easily available and more cost-effective than PET tracers. Nevertheless, the high sensitivity of PET tracers and their integration as biomarkers with multi-capability applications makes them very well suited for small animal imaging. Tracers such as ${ }^{18} \mathrm{~F}-\mathrm{FDG}$, a glucose analogue, and ${ }^{18} \mathrm{~F}$-FLT, a pyrimidine analogue are used as biomarkers of tissue metabolic activity and inflammation, and cell proliferation, respectively.

\section{PET/SPECT imaging applications}

Several studies have successfully reported the use of Tc99 m-labelled diphosphonate compounds, e.g. methylene diphosphonate (MDP), hydroxymethane diphosphonate and hydroxyethylidene diphosphonate, to detect changes in bone turnover and cartilage composition in osteoarthritis models in rodents [47]. The co-registration of micro-SPECT/micro-CT images allows the detection of high Tc-99 m MDP uptake, depicting areas of high bone turnover, e.g. joints (knees, shoulders), spine and skull (Figure 5). SPECT imaging of articular cartilage in rodents has been reported using $\mathrm{N}$-[triethylamonium]-3propyl-[15] ane-N (NTP) bound to Tc-99 $\mathrm{m}$. NTP has a high affinity for binding to negatively charged sulphate groups of the glycosaminoglycans, a major component of the cartilage matrix, and it has been successfully used for in vivo imaging of a cartilage tumoural model in rats [48].

For PET imaging, ${ }^{18} \mathrm{~F}$-fluoride $(\mathrm{NaF})$ is being extensively used for assessing bone metabolism, and it has a similar uptake mechanism to $99 \mathrm{~m}^{\mathrm{m}} \mathrm{T}$-MDP, being absorbed onto bone surfaces. This tracer diffuses well through capillaries reaching bone extracellular fluid where it is rapidly incorporated in the bone hydroxyapatite. Its negligible binding to plasma proteins, rapid blood and renal clearance with the high uptake after injection (40 to $60 \mathrm{~min}$ ) provides a higher accuracy than SPECT imaging. Similar to MDP, it is taken up preferentially in malignant cancerous bone lesions such as sclerotic metastases and areas of altered osteogenic activity, reflecting an increase in regional blood flow and bone turnover [49] and for detecting bone microdamage [50] in preclinical models.

${ }^{18} \mathrm{~F}$-FDG is another PET tracer that can be used to assess inflammatory activity indirectly in bone. ${ }^{18} \mathrm{~F}$-FDG is not specifically targeted to bone, but as a glucose analogue, it provides a sensitive complementary functional biomarker in defining areas of inflammation in the axial and appendicular skeleton. PET scanning with ${ }^{18} \mathrm{~F}$-FDG has great potential for assessing fracture healing as it can provide a direct quantitative non-invasive assessment of the metabolic activity in the region of interest and therefore measure bone repair in fracture models [51], providing information on the treatment and prognosis of delayed fracture healing. ${ }^{18} \mathrm{~F}$-FDG is also widely used in clinical oncology in musculoskeletal sarcomas, as the tracer is directly taken up into tumour cells and is often used to detect metastases. It has been used successfully to localise and quantify skeletal metabolic activity in the study of preclinical cancer metastasis in bone [52].

\section{Optical imaging technologies}

Optical imaging, including fluorescence and bioluminescence, is becoming an attractive tool for examining and monitoring disease states and to determine therapy effectiveness in living tissues in preclinical models.

\section{Fluorescence technology and applications}

Fluorescence imaging relies on the detection of light emission of specific fluorophore when excited by appropriate wavelength energy. Several approaches have been described in vivo, including the use of non-specific dye tracking (e.g. for detection of non-specific inflammatory 


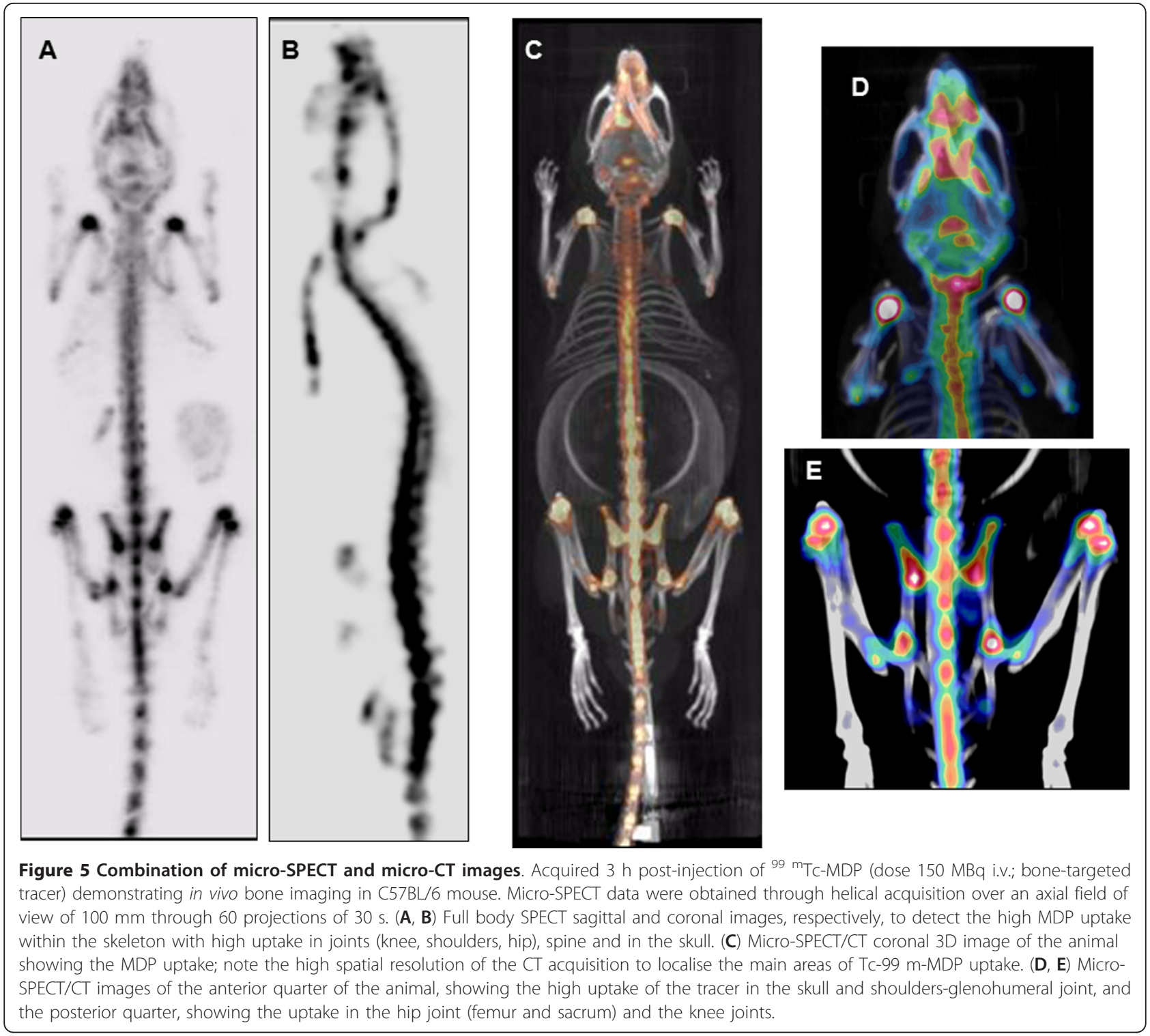

sites), the use of fluorophores tagged-antibody-based targets for specific cell molecules and/or metabolic pathways and the development of fluorescence reporter proteins (e.g. GFP) for the detection of gene expression. Targeted fluorescence imaging of arthritic joints has shown an increase in fluorescence signal in the arthritic joints in disease models. This is in part associated with increased blood perfusion and vascular leakiness, which are recognised to occur in areas of inflamed synovium [53]. In vivo targeting of specific key components identified during the inflammatory process such as the labelling of the F4/80 antigen on macrophages has been achieved [54]. Similarly, other components of the inflammatory cascade can be traced in vivo. For example, E-selectin-targeted in vivo imaging is a quantifiable method of detecting endothelial activation. E-selectin or endothelial adhesion molecule- 1 is a $115-\mathrm{kDa}$ glycoprotein induced on endothelial cells in response to proinflammatory cytokines involved in rheumatoid arthritis such as interleukin- $1 \beta$ and tumour necrosis factor alpha (TNF- $\alpha$ ) [55]. E-selectin has been well validated as a potential biomarker of disease activity in rheumatoid arthritis [56].

Anti-E-selectin antibody labelled with NIR fluorophore has demonstrated specific signal increases compared to control antibody in a mouse model of paw swelling induced by the intra-plantar injection of TNF- $\alpha$. This has also been demonstrated in acute collagen-induced arthritis, a widely used model of RA. Mapping of fluorescent E-selectin-specific signal in difference to the signal returned from fluorescently labelled control antibody is demonstrated in Figure 6[57]. Utilising 

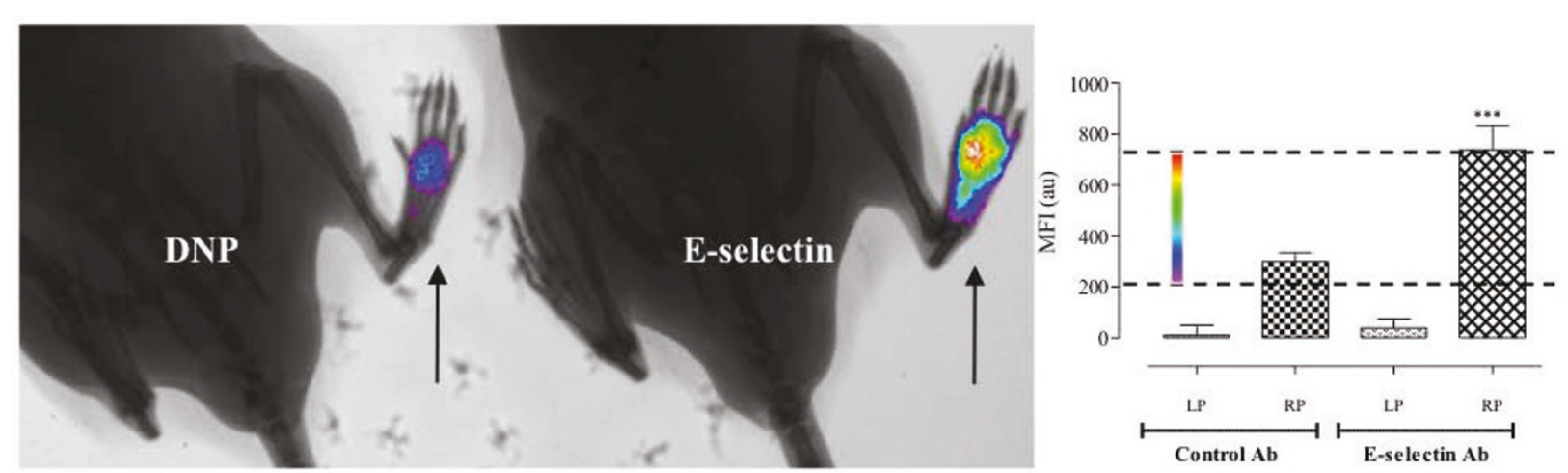

Figure 6 Specific anti-E-selectin targeted fluorescent signal co-registered with X-ray imaging. Following injection of either anti-E-selectin or anti-DNP (control) antibodies labelled with Dylight $750 \mathrm{~nm}$ NIR fluorophore (Thermo Fisher Scientific Inc., Rockford, IL, USA) at a dose of $5 \mu \mathrm{g}$ i.v., paw swelling was induced by intraplantar injection of murine TNF- $\alpha$ into the right paw (RP) in C57/BL6 mice (marked by arrows) ( $n=4$ to 6). Mean fluorescence signal (MFI) quantified at the $8 \mathrm{~h}$ time point is shown for different groups of mice. The mean background intensity from control and anti-E-selectin-targeted animals was subtracted. In the left-hand panel, the corresponding fluorescent image overlaid onto a coregistered $\mathrm{X}$-ray. The colour wheel to depict signal intensity has been adjusted to the range as shown on the graph.

fluorescent compounds to label antibodies allows for a more targeted and specific approach to identify different components of disease, quantify levels of inflammation and assess the effect of novel therapies. Similarly, activity-based fluorophores where signal becomes amplified at the site of inflammation have also been used successfully in mouse models of arthritis demonstrating increases in signal intensity in the injured joint [58]. This type of approach may be particularly useful for optical imaging in OA, since local perturbations in proteolytic activity may be small and require amplification for adequate signal detection.

Fluorescence is also extensively used to image the skeleton, with a multitude of commercially available bone specific fluorophores (e.g. OsteoSense ${ }^{\mathrm{TM}}$ from PerkinElmer, Waltham, MA, USA), fluorescently labelled alendronate from Caliper ${ }^{\circledR}$ LifeSciences (Hopkinton, MA, USA) and BoneTag ${ }^{\mathrm{TM}}$ from LI-COR ${ }^{\circledR}$ Biosciences (Lincoln, NE, USA) [59], which are incorporated in the calcified bone matrix at spots with high bone turnover and are therefore good indicators of bone remodelling in sites of bone damage such as fractures and cancerinduced osteolytic/blastic lesions [60].

\section{Bioluminescence technology and applications}

Bioluminescence imaging is based on the detection of photons produced after an enzymatic reaction in living organisms. It is commonly used in reporter gene assays, where the promoters of genes under study have been linked to the luciferase gene, which in the presence of its substrate (luciferin) generates light. The most commonly used luciferase enzyme used for bioluminescence imaging purposes comes from the firefly (emitting light around $560 \mathrm{~nm}$ ). This approach requires the luciferase enzyme to be transfected into the living cells and must be tightly controlled with the appropriate promoter. Also, a substrate needs to be delivered where the enzyme may be expressed. This may be challenging for certain organs and may affect the dynamics of the emission response due to different pharmacokinetic profiles related to altered tissue properties. Despite these conditions, its high specificity, low background signal, high signal-to-noise ratio and the ability to study gene expression over the lifetime of the animal are reasons why this technology is used extensively for in vivo imaging studies. Bioluminescence imaging has been successfully utilised to study the development of arthritis in transgenic mouse models [61], and because of its high sensitivity, it is also used widely in bone metastasis studies, to monitor the development and progression of luciferase positive tumour cell lines within the whole body [62].

\section{Challenges for in vivo preclinical imaging}

The implementation of non-invasive imaging technologies provides a very useful avenue for more rapid, efficacious and cost-effective use and characterisation of animal disease models. They represent a good alternative assessment tool for the invasive techniques and histological or biochemical assays that are already used extensively. By combining different imaging modalities, accurate quantitative and qualitative structural and functional data targeting molecular mechanisms can be acquired (Table 1).

The ability to image live animals is one of the most important advantages of these technologies. But this also represents a major challenge as biological motion, not only gross body movement but also that induced by breathing and cardiac activity, affects the resolution of 
Table 1 Main features of current imaging modalities for in vivo preclinical musculoskeletal research

\begin{tabular}{|c|c|c|c|c|c|c|c|}
\hline $\begin{array}{l}\text { Imaging } \\
\text { modality }\end{array}$ & Resolution & Sensitivity & $\begin{array}{l}\text { Imaging } \\
\text { time }\end{array}$ & Application & Radiation & $\begin{array}{l}\text { Detection } \\
\text { depth of } \\
\text { view }\end{array}$ & Limitations \\
\hline \multirow[t]{2}{*}{ Micro-CT } & $10 \mu \mathrm{m}$ & m-cmol & $\begin{array}{l}\text { Seconds } \\
\text { to } \\
\text { minutes }\end{array}$ & $\begin{array}{l}\text { Excellent contrast for mineralized } \\
\text { tissue }\end{array}$ & X-rays & No limit & Radiation exposure \\
\hline & & & & Mostly anatomical imaging & & & Long acquisition times \\
\hline \multirow[t]{3}{*}{ MRI } & $\begin{array}{l}50 \text { to } 100 \\
\mu \mathrm{m}\end{array}$ & $\mu-\mathrm{mmol}$ & $\begin{array}{l}\text { Minutes } \\
\text { to hours }\end{array}$ & $\begin{array}{c}\text { Excellent contrast tissue resolution } \\
\text {-anatomical and functional } \\
\text { Imaging }\end{array}$ & No & No limit & $\begin{array}{l}\text { High investment } \\
\text { infrastructure and running } \\
\text { cost }\end{array}$ \\
\hline & & & & & & & Long acquisition time \\
\hline & & & & & & & Expert operator \\
\hline \multirow[t]{3}{*}{ PET } & 1 to $2 \mathrm{~mm}$ & $\mathrm{p}-\mathrm{nmol}$ & Minutes & Functional imaging & $\begin{array}{l}\text { Gamma } \\
\text { radiation }\end{array}$ & No limit & $\begin{array}{l}\text { Short half-life PET tracer } \\
\text { (requirement cyclotron } \\
\text { unit) }\end{array}$ \\
\hline & & & & & & & High cost of tracers \\
\hline & & & & & & & $\begin{array}{l}\text { High investment } \\
\text { infrastructure }\end{array}$ \\
\hline \multirow[t]{2}{*}{ SPECT } & $<1 \mathrm{~mm}$ & $\mathrm{p}$-nmol & Minutes & Tomographic functional imaging & $\begin{array}{l}\text { Gamma } \\
\text { radiation }\end{array}$ & No limit & Limited sensitivity \\
\hline & & & & & & & $\begin{array}{l}\text { High investment } \\
\text { infrastructure }\end{array}$ \\
\hline $\begin{array}{l}\text { Fluorescent } \\
\text { (optical imaging) }\end{array}$ & 1 to $2 \mathrm{~mm}$ & p-nmol & $\begin{array}{l}\text { Seconds } \\
\text { to } \\
\text { minutes }\end{array}$ & Functional imaging & $\begin{array}{l}\text { Fluorescent } \\
\text { emission }\end{array}$ & $<1$ to $10 \mathrm{~cm}$ & $\begin{array}{l}\text { Not translated into clinical } \\
\text { modality }\end{array}$ \\
\hline \multirow[t]{2}{*}{$\begin{array}{l}\text { Bioluminescence } \\
\text { (optical imaging) }\end{array}$} & 1 to $2 \mathrm{~mm}$ & p-nmol & $\begin{array}{l}\text { Seconds } \\
\text { to } \\
\text { minutes }\end{array}$ & Functional imaging & $\begin{array}{l}\text { Light } \\
\text { emission }\end{array}$ & $<10 \mathrm{~cm}$ & $\begin{array}{l}\text { Not translated into clinical } \\
\text { modality }\end{array}$ \\
\hline & & & & & & & Injection of substrate \\
\hline
\end{tabular}

the images. Animals are imaged under anaesthesia, which helps to restrain their gross motion, but there is still the need to control cardiac and respiratory motion. Moreover, anaesthesia has undesirable effects interfering with the body temperature control and also respiratory rates. Close monitoring is required for refining the efficacy of preclinical testing and ensuring the standardisation and repeatability of studies.

Another important challenge is the small size of animal specimens that require high resolution and sensitivity in smaller fields of view and also take into account the physiological motions of the animals. The implementation of gating methods has markedly improved the acquisitions, minimising any interference effects due to the physiological movement. Gating (prospective or retrospective, if image acquisition is acquired simultaneously or processed post-acquisition) is already implemented in most of the preclinical in vivo imaging systems using specialised software. The benefits of applying gating during image acquisition have been well reported in micro-CT imaging of rodents [13]. While such motion artefacts may not be so relevant when imaging appendicular skeleton, their effects are significant when imaging the skull and axial skeleton.

Post-image processing is also of paramount importance, especially when integrating data acquired through different modalities and to correct for the posture and shape variability between different animals or within the same animal through a follow-up study. While analysing techniques used for these multidimensional image datasets are quite complex and are not easily interchangeable, many software tools have been developed (e.g. Amira, ImageJ, OsiriX; see review [63]) providing facilities for image registration, 3D surface-rendering and image segmentation. To integrate different datasets, the most commonly used technique is atlas registration, whereby individual acquisitions are registered to an idealised expert-defined atlas based on prior images. Recently, Baiker and collaborators [64] developed a fully automated method for atlas-based whole-body segmentation from low-contrast micro-CT data which effectively allowed intra- and inter-subject registrations using data acquired in vivo, with remarkable accuracy, overcoming large variations in posture and shape. Snoeks and collaborators [65] nicely demonstrated the strength of multi-modality imaging by combining the use of bioluminescence and micro-CT to image bone metastases in vivo.

With the increasing demand to acquire higher temporal and spatial resolution images, animal welfare is an important consideration, especially the cumulative effect of any radiation dose on the animal's well-being and 
physiological status. This is of most concern in longitudinal studies where the cumulative radiation dose can be extremely high. The effect of ionizing radiation in rodents has been well studied after micro-CT. The lethal dose in mice, which is typically expressed as $\mathrm{LD}_{50 / 30}$ (whole-body radiation dose that would kill $50 \%$ of exposed animals within 30 days) is in the range 5 to 7.6 Gy. The typical X-ray whole-body radiation dose for a 3D micro-CT scan ranges from 0.017 to 0.78 Gy [4]. These sub-lethal doses of radiation are unlikely to really compromise the animal using in vivo micro-CT for longitudinal studies. In the case of musculoskeletal applications, higher resolutions may be required, but it is likely that only a small area of the animal will be imaged. The exposure to radiation may be an issue to be considered in oncological in vivo studies as the dose is perhaps more likely to have an effect on the growth of the tumours to be imaged [66] and also could influence other biological pathways affecting bone remodelling [67].

The potential of small animal imaging goes far beyond anatomical studies, and by combining all the different imaging modalities, functional and anatomical analyses can be integrated. While PET and SPECT can provide excellent sensitivity for functional procedures through labelled biomarkers, combination with $\mathrm{CT}$ imaging allows for accurate spatial correlation of the biomarker within the body. Similarly, other multi-modality approaches such as PET/MRI are under development to increase the spatial and temporal resolution of the PET and the sensitivity of MRI [68].

With the increasing availability of new tracers as biomarkers to target specific musculoskeletal disorders, data from PET/SPECT or optical imaging combined with CT will play a key role in translational bone and cartilage research. Similarly, optimization of the micro-CT system is likely to lead to more sensitive detectors and will provide higher spatial resolution with shorter acquisition times, reduced radiation dose with improvements in gating acquisitions and interactive reconstruction. These will undoubtedly reinforce the use of these techniques for preclinical research into bone and cartilage disorders.

\section{Conclusion}

In conclusion, the increasing use of imaging technologies in animal models supports its prominent role in translational research for bone and joint disorders. With ongoing developments in micro-CT and MRI preclinical scanners, imaging of bone and cartilage structures can be achieved down to the micro-structural level, effectively visualising trabecular bone structure and cartilage composition. On the other hand, the implementation of PET and SPECT and optical imaging in the musculoskeletal preclinical field provides significant potential for investigating the dynamics of bone and joint biological processes at the molecular and cellular levels with the ability to monitor the effectiveness of novel therapeutic targets. New PET and fluorescent tracers are being developed as biomarkers for specific disorders that can be utilised for testing the efficacy of new drugs and validating their safety at early stages of drug development. Ongoing research is focussing on integrating different imaging modalities with the aim of facilitating successful translational applications from anatomical to functional endpoints, to improve the efficacy in musculoskeletal preclinical studies.

\section{Author details}

${ }^{1}$ Biological Imaging Centre (BIC), Medical Research Council (MRC) Clinical Sciences Centre, Imperial College London, Hammersmith Campus, London W12 ONN, UK ${ }^{2}$ Faculty of Medicine, Kennedy Institute of Rheumatology, Imperial College London, London W6 8LH, UK

\section{Authors' contributions}

JLT performed some of the reported animal experiments, reviewed current literature, provided the concept for the review and wrote the manuscript.

MK helped in the imaging experiments, provided valuable intellectual advice on the imaging technology and critically reviewed the manuscript. LLG provided valuable input in the fluorescence technology and critically edited the manuscript. TV provided some of the tissue samples for the study, gave valuable intellectual input particularly on joint disorders and critically reviewed the manuscript. MWA and WG critically reviewed the manuscript and supported the conception of this review. All the authors read and approved the final manuscript.

\section{Competing interests}

The authors declare that they have no competing interests.

Received: 8 April 2011 Accepted: 29 July 2011 Published: 29 July 2011

\section{References}

1. European Science Foundation: Rheumatic Disease-a Major Challenge for European Research and Health Care. European Science Foundation Policy Briefing 2006, 6:1-4.

2. Vanderheyden $\mathrm{J}$ : The use of imaging in preclinical drug development. $Q$ J Nucl Med Mol Imaging 2009, 53:374-381.

3. Schambach SJ, Bag S, Schilling L, Gronden C, Brockmann MA: Application of micro-CT in small animal imaging. Methods 2010, 50:2-13.

4. Badea $C T$, Drangova $M$, Holdsworth DW, Johnson GA: In vivo small animal imaging using micro-CT and digital substraction angiography. Phys Med Biol 2008, 53:319-350.

5. Holdsworth DW, Thornton M: Micro-CT in small animal and specimen imaging. Trends in Biotechnology 2002, 20:34-39.

6. Winkelmann $C T$, Wise LD: High-throughput micro-computed tomography imaging as a method to evaluate rat and rabbit fetal skeletal abnormalities for developmental toxicity studies. J Pharmacol Toxicol Methods 2009, 59:156-165.

7. Bouxsein ML, Boyd SK, Christiansen BA, Guldberg RE, Jepsen KJ, Müller R: Guidelines for assessment of bone microstructure in rodents using micro-computed tomography. J Bone Miner Res 2010, 25:1468-1486.

8. Feldkamp LA, Goldstein SA, Parfitt AM, Jesion G, Kleerekoper M: The direct examination of three-dimensional bone architecture in vitro by computed tomography. J Bone Miner Res 1989, 4:3-11.

9. Rüegsegger P, Koller B, Müller R: A microtomographic system for the nondestructive evaluation of bone architecture. Calcif Tissue Int 1996, 58:24-29.

10. Wachsmuth L, Engelke K: High-resolution imaging of osteoarthritis using microcomputed tomography. Methods Mol Med 2004, 101:231-248.

11. Feldkamp LA, Davis LC, Kress LW: Practical cone-beam algorithm. J Opt Soc Am 1984, 1:612-619. 
12. Du LY, Umoh J, Nikolov HN, Pollmann SI, Lee TY, Holdsworth DW: A quality assurance phantom for the performance evaluation of volumetric microCT systems. Phys Med Biol 2007, 52:7087-7108.

13. Taschereau R, Silverman RW, Chatzijoannou AF: Dual-energy attenuation coefficient decomposition with differential filtration and application to a micro-CT scanner. Phys Med Biol 2010, 55:1141-1155.

14. Cherry SR: Multimodality imaging: beyond PET/CT and SPECT/CT. Semin Nucl Med 2009, 39:348-353.

15. Badea C, Hedlund LW, Johnson GA: Micro-CT with respiratory and cardiac gating. Med Phys 2004, 31:3324-3329.

16. Morgan EF, Mason ZD, Chien KB, Pfeiffer AJ, Barnes GL, Einhorn TA, Gerstenfeld LC: Micro-computed tomography assessment of fracture healing: relationships among callus structure, composition, and mechanical function. Bone 2009, 44:335-344.

17. Freeman TA, Patel P, Parvizi J, Antoci V Jr, Shapiro IM: Micro-CT analysis with multiple thresholds allows detection of bone formation and resorption during ultrasound-treated fracture healing. J Orthop Res 2009, 27:673-679.

18. Johnson LC, Johnson RW, Munoz SA, Mundy GR, Peterson TE, Sterling JA: Longitudinal live animal microCT allows for quantitative analysis of tumor-induced bone destruction. Bone 2011, 48:141-151.

19. Campell GM, Ominsky MS, Boyd SK: Bone quality in partially recovered after the discontinuation of RANKL administration in rats by increased bone mass on existing trabeculae: an in vivo micro-Ct study. Osteoporos Int 2011, 22:931-942.

20. Kristensen E, Parsons TE, Hallgrímsson B, Boyd SK: A novel 3-D imagebased morphological method for phenotypic analysis. IEEE Trans Biomed Eng 2008, 55:2826-2831.

21. Ritman EL: Small-animal CT: its difference from, and impact on, clinical CT. Nucl Instrum Methods Phys Res A 2007, 580:968-970.

22. Boyd SK, Davison P, Müller R, Gasser JA: Monitoring individual morphological changes over time in ovariectomized rats by in vivo micro-computed tomography. Bone 2006, 39:854-862.

23. Klinck RJ, Campbell GM, Boyd SK: Radiation effects on bone architecture in mice and rats resulting from in vivo micro-computed tomography scanning. Med Eng Phys 2008, 30:888-895.

24. Barck KH, Lee WP, Diehl LJ, Ross J, Gribling P, Zhang Y, Nguyen K, van Bruggen N, Hurst S, Carano RA: Quantification of cortical bone loss and repair for therapeutic evaluation in collagen-induced arthritis, by microcomputed tomography and automated image analysis. Arthritis Rheum 2004, 50:3377-3386.

25. Piscaer TM, Waarsing JH, Kops N, Pavljasevic P, Verhaar JA, van Osch GJ, Weinans $\mathrm{H}$ : In vivo imaging of cartilage degeneration using microCTarthrography. Osteoarthritis Cartilage 2008, 16:1011-1017.

26. Piscaer TM, van Osch GJVM, Verharra JAN, Weinans H: Imaging of experimental osteoarthritis in small animal models. Biorheology 2008, 45:355-364.

27. Botter SM, Osch GJVM, Waarsinge JH, van der Linden JC, Verhaar JAN, Pols HAP, van Leeuwen JPTM, Weinans H: Cartilage damage pattern in relation to subchondral plate thickness in a collagenase-induced model of osteoarthritis. Osteoarthritis Cartilage 2008, 16:506-514.

28. Moodie JP, Stok KS, Muller R, Vincent TL, Shefelbine SJ: Multimodal imaging demonstrates concomitant changes in bone and cartilage after destabilization of the medial meniscus and increased joint laxity. Osteoarthritis Cartilage 2011, 19:163-170.

29. Stok KS, Pelled G, Zilberam Y, Kallai I, Goldhahn, Gazit D, Muller R: Revealing the interplay of bone and cartilage in osteoarthritis through multimodal imaging of murine joints. Bone 2009, 45:414-422.

30. Botter SM, van Osch GJ, Waarsing JH, Day JS, Verhaar JA, Pols HA, van Leeuwen JP, Weinans H: Quantification of subchondral bone changes in murine osteorathritis model using micro-CT. Biorheology 2006, 43:379-388.

31. Mc Erlain DD, Appleton CT, Litchfield RB, Pitelka V, Henry JL, Bernier SM, Beier F, Holdsworth DW: Study of subchondral bone adaptations in a rodent surgical model of OA using in vivo micro-computed tomography. Osteoarthritis Cartilage 2008, 16:458-469.

32. Roemer FW, Mohr A, Lynch JA, Meta MD, Guermazi A, Genant HK: Micro-CT arthrography: a pilot study for the ex vivo visualization of the rat knee joint. AJR Am J Roentgenol 2005, 184:1215-1219.

33. Palmer AW, Guldberg RE, Levbenston ME: Analysis of cartilage matrix fixed charge density qand three-dimensional morphology via contrast- enhanced microcomputed tomography. Proc Natl Acad Sci USA 2006, 103:19255-19260.

34. Blair-Levy JM, Watts CE, Fiorientino NM, Dimitriadis EK, Marinie JC, Lipsky PE: A type I Collagen defect leads to rapidly progressive osteoarthritis in a mouse model. Arthritis \& Rheumatism 58:1096-1106.

35. Faure $P$, Doan BT, Beloil JC: In vivo high resolution three-dimensional MRI studies of rat joints at 7T. NMR in Biomedicine 2003, 16:484-493.

36. Laurent D, O'Byrne E, Wasvary J, Pellas TC: In vivo MRI of cartilage pathogenesis in surgical models of osteoarthritis. Skeletal Radiol 2006, 35:555-564.

37. Jiang Y, Zhao J, White DL, Genant HK: Micro Ct and Micro MRI imaging of 3D architecture of animal skeleton. J Musculoskel Neuron Intercat 2000, 1:45-51.

38. Wang HH, Wang YX, Griffith JF, Sun YL, Zhang G, Chan CW, Qin L, Ahuja AT, Teng LS: Pitfalls in interpreting rat knee joint magnetic resonance images and their histological correlation. Acta Radiol 2009, 50:1042-1048.

39. Takahashi M, Wehrli FW, Hilaire L, Zemel BS, Hwang SN: In vivo NMR microscopy allows short-term serial assessment of multiple skeletal implications of corticosteroid exposure. Proc Natl Acad Sci USA 2002, 99:4574-4579.

40. Anumula S, Wehrli SL, Magland J, Wright AC, Wehrli FW: Ultra-short echotime MRI detects changes in bone mineralization and water content in OVX rat bone in response to alendronate treatment. Bone 2010, 46:1391-1399.

41. Goebel JC, Bolbos R, Pham M, Galois L, Rengle A, Loeuille D, Netter P, Gillet $P$, Beuf $O$, Watrin-Pinzano A: In vivo high-resolution MRI (7T) of femoro-tibial cartilage changes in the rat anterior cruciate ligament transection model of osteoarthritis: a cross-sectional study. Rheumatology (Oxford) 2010, 49:1654-1664.

42. Spandonis $Y$, Heese FP, Hall LD: High resolution MRI relaxation measurements of water in the articular cartilage of the meniscectomized rat knee at 4.7 T. Magn Reson Imaging 2004, 22:943-951.

43. Liu S, Shen S, Zhu T, Liang W, Huang L, Chen H, Wu H: Gadoliniumenhanced magnetic resonance imaging of the knee: an experimental approach. Skeletal Radiol 2010, 39:885-890

44. Rengle A, Armenean M, Bolbos R, Goebel JC, Pinzano-Watrin A, SaintJalmes $H$, Gillet $P$, Beauf $O$ : A dedicated two-channel phased-array receiver coild for high-resolution $\mathrm{MRI}$ of the rat knee cartilage at 7T. IEEE Transactions on Biomedical Engineering 2009, 56:2891-2897.

45. Riemann B, Schäfers KP, Schober O, Schäfers M: Small animal PET in preclinical studies: opportunities and challenges. QJ NuCl Med Mil Imaging 2008, 52:215-221.

46. Franc BJ, Acton PD, Mari C, Hasegawa BH: Small Animal SPECT and SPECT/ CT: Important tools for preclinical investigation. J NuCl Med 2008, 49:1651-1663.

47. Miot-Noirault E, Vidal A, Auzeloux P, Madelmont JC, Maublant J, Moins N: First in vivo SPECT imaging of mouse femorotibial cartilage using $99 \mathrm{~m}$ TC-NTP 15-5. Mol Imaging 2008, 7:263-271.

48. Miot-Noirault E, Gouin F, Vidal A, Rapp M, Maublant J, Askienazy S, Chezal JM, Heymann D, Redini F, Moins N: First preclinical imaging of primary cartilage neoplasm and its local recurrence using ${ }^{99} \mathrm{~m}$ Tc-NTP 15-5 radiotracer. J Nucl Med 2009, 50:1541-1547.

49. Blake GM, Park-Holohan SJ, Cook GJ, Fogelman I: Quantitative studies of bone with the use of $18 \mathrm{~F}$-fluoride and ${ }^{99} \mathrm{~m} T \mathrm{~T}$-methylene diphosphonate. Semin Nucl Med 2001, 31:28-49.

50. Li J, Miller MA, Hutchins GD, Burr DB: Imaging bone microdamage in vivo with positron emission tomography. Bone 2005, 37:819-824.

51. Hsu WK, Feeley BT, Krenek L, Stout DB, Chatzijoannou AF, Lieberman JR: The use of $18 \mathrm{~F}$-fluoride and 18F-FDG PET scans to assess fracture healing in a rat femur model. Eur J Nucl Med Mol Imaging 2007, 34:1291-1301.

52. Hsu WK, Virk MS, Feeley BT, Stout DB, Chatzijoannou AF, Lieberman JR: Characterisation of osteolytic, osteoblastic and mixed lesions in a prostate cancer mouse model using 18F-FDG and 18F-fluoride PET/CT. J Nucl Med 2008, 49:414-442.

53. Andersson SE, Johansson A, Lexmüller K, Ekström GM: Physiological characterization of $\mathrm{mBSA}$ antigen induced arthritis in the rat. II. Joint blood flow, glucose metabolism, and cell proliferation. J Rheumatol 1998, 25:1778-1784. 
54. Hansch A, Frey O, Hilger I, Sauner D, Haas M, Schmidt D, Kurrat C, Gajda M, Malich A, Bräuer R, Kaiser WA: In vivo imaging of experimental arthritis with near-infrared fluorescence. Arthritis Rheum 2004, 50:626-632.

55. Wellicome SM, Thornhill MH, Pitzalis C, Thomas DS, Lanchbury JS, Panayi GS, Haskard DO: A monoclonal antibody that detects a novel antigen on endothelial cells that is induced by tumor necrosis factor, il1, or lipopolysaccharide. J Immunol 1990, 144:2558-2565.

56. Jamar F, Houssiau FA, Devogelaer JP, Chapman PT, Haskard DO, Beaujean V, Beckers C, Manicourt DH, Peters AM: Scintigraphy using a technetium 99 m-labelled anti-e-selectin fab fragment in rheumatoid arthritis. Rheumatology (Oxford) 2002, 41:53-61.

57. Gompels LL, Madden L, Han Lim N, Inglis JJ, McConnell E, Vincent TL, Haskard DO, Paleolog EM: In vivo fluorescence imaging of E-selectin: quantitative detection of endothelial activation in arthritis. Arthritis \& Rheumatism 2011, 63(1):107-117.

58. Lai WF, Chang CH, Tang Y, Bronson R, Tung CH: Early diagnosis of osteoarthritis using cathepsin B sensitive near-infrared fluorescent probes. Osteoarthritis Cartilage 2004, 12:239-244.

59. Snoeks TJ, Khmelinskii A, Lelieveldt BP, Kaijzel EL, Löwik CW: Optical advances in skeletal imaging applied to bone metastases. Bone 2011, 48:106-114.

60. Zaheer A, Lenkinski RE, Mahmood A, Jones AG, Cantley LC, Frangioni JV: In vivo near-infrared fluorescence imaging of osteoblastic activity. Nat Biotechnol 2001, 19:1148-1154.

61. Carlsen H, Moskaug JØ, Fromm SH, Blomhoff R: In vivo imaging of NFkappa B activity. J Immunol 2002, 168:1441-1446.

62. Wetterwald A, van der Pluijm G, Que I, Sijmons B, Buijs J, Karperien M, Löwik CW, Gautschi E, Thalmann GN, Cecchini MG: Optical imaging of cancer metastasis to bone marrow: a mouse model of minimal residual disease. Am J Pathol 2002, 160:1143-1153.

63. Walter T, Shattuck DW, Baldock R, Bastin ME, Carpenter AE, Duce S, Ellenberg J, Fraser A, Hamilton N, Pieper S, Ragan MA, Schneider JE, Tomancak P, Heriche JK: Visualization of image data from cells to organisms. Nat Methods 2010, 7:526-541.

64. Baiker M, Milles J, Dijkstra J, Henning TD, Weber AW, Que I, Kaijzel EL, Löwik CWGM, Reiber JHC, Lelieveldt BPF: Atlas-based whole-body segmentation of mice from low-contrast Micro-CT data. Medical Image Analysis 2010, 14:723-737.

65. Snoeks TJA, Khmelinskii A, lelieveldt BPF, Kaijzel EL, Löwik CWGM: Optical advances in skeletal imaging applied to bone metastases. Bone 2011, 48:106-114.

66. Bhattacharjee $D$, Ito $A$ : Deceleration of carcinogenic potential by adaptation with low dose gamma irradiation. In Vivo 2001, 15:87-92.

67. Boone JM, Velazquez O, Cherry SR: Small-animal X-ray dose from microCT. Mol Imaging 2004, 3:149-158.

68. de Kemp RA, Epstein FH, Catana C, Tsui BM, Ritman EL: Small-animal molecular imaging methods. J Nucl Med 2010, 51(Suppl 1):185-32S.

doi:10.1186/2191-219X-1-11

Cite this article as: Tremoleda et al: Imaging technologies for preclinical models of bone and joint disorders. EJNMMI Research 2011 1:11.

\section{Submit your manuscript to a SpringerOpen ${ }^{\mathcal{O}}$ journal and benefit from:}

- Convenient online submission

- Rigorous peer review

- Immediate publication on acceptance

- Open access: articles freely available online

- High visibility within the field

- Retaining the copyright to your article

Submit your next manuscript at $\gg$ springeropen.com 